\title{
Intuitive Tip of the Tongue Judgments Predict Subsequent Problem Solving One Day Later
}

\author{
Azurii K. Collier ${ }^{1}$ and Mark Beeman ${ }^{1}$
}

\begin{abstract}
:
Often when failing to solve problems, individuals report some idea of the solution, but cannot explicitly access the idea. We investigated whether such intuition would relate to improvements in solving and to the manner in which a problem was solved after a 24- hour delay. On Day 1, participants attempted to solve Compound Remote Associate problems, for which they viewed three problem words (crab, sauce, pine) and tried to generate one solution word (apple) that could form a compound word with each problem word (crabapple, applesauce, pineapple). For problems they failed to solve, participants reported whether they had an intuitive sense that they might have solution related processing in the back of their mind, similar to a Tip-of-the-Tongue (TOT) experience. After an overnight delay, on Day 2 participants attempted to solve unsolved Old problems from Day 1 (mixed among New problems). Participants solved more Old problems for which they reported a TOT on Day 1 than Old problems without a TOT, demonstrating a TOT specific incubation effect. Interestingly, participants reported solving a marginally higher proportion of these TOT problems, compared to No TOT problems, with insight. Results suggest that intuitive TOT judgments are indicative of subthreshold solution related activation that can facilitate eventual problem solving, especially with insight.
\end{abstract}

Keywords:

insight, problem solving, intuition

\footnotetext{
${ }^{1}$ Northwestern University
} 


\section{Introduction}

Intuition refers to the ability to make a judgment about stimulus properties without being able to refer consciously to the knowledge underlying the judgment (Ilg et al., 2007). Some empirical studies have demonstrated the reliability of intuitive judgments on lower level perceptual stimuli; fewer studies have demonstrated the reliability of intuitive judgments in relation to higher order cognitive processes, like problem solving (Bowers, Regehr, Balthazard, \& Parker, 1990; Bolte \& Goschke, 2005; Bolte, Goschke \& Kuhl, 2003). Still less is known about whether, and how, initial intuitive judgments relate to eventual problem solution. The current experiment investigated whether intuitive Tip of the Tongue (TOT) judgments would reliably predict which unsolved word problems are more likely to be solved one day later, during a second attempt at problem solving.

Problem solving can be characterized as a search through a mental problem space aimed at generating a solution (Newell \& Simon, 1972). However, individuals are often unable to generate a solution during the first attempt at problem solving. A failed attempt may partially activate the solution and solution related information, thereby making the solution more accessible during subsequent attempts at problem solving (Connor, Balota, \& Neely, 1992; Seifert \& Patalano, 1991). It would be advantageous for solvers to be sensitive to which unsolved problems are likely to be solvable during a subsequent attempt at problem solving.

There is evidence in the metacognition and memory literatures suggesting that individuals are sensitive to which unrecalled items are more likely to be recalled during a subsequent attempt at recall. TOT experiences occur when the subthreshold activation of an unrecalled target word is strong enough to elicit a metacognitive dissociation, but not strong enough to elicit complete recall of the target word (Brown \& McNeill, 1966; Maril, Wagner, \& Schacter, 2001). That is, people may be aware of the concept, and even of some aspects of the words' phonology, but cannot access the full word. An unrecalled item that elicits a TOT represents higher subthreshold activation and is more likely to be recalled compared to an unrecalled item that elicits no TOT (Schwartz, 1999; 2011).

We used a slightly different type of TOT judgment for problem solving to investigate whether these judgments are related to subthreshold solution related activation during an initial failed attempt at problem solving and possibly predict improvements in problem solving following a break in conscious work. In our study, a TOT judgment indicated that participants intuited that they were "on to something" or had "the solution in there somewhere," but the concept itself (let alone the lexical item) still eluded conscious access. When individuals are unable to solve a problem, they may experience an intuitive TOT suggesting that the solution, though not yet retrieved, is being processed. The TOT judgment may signal which problems have solutions activated at a subthreshold level, thus which problems are more likely to be solved during a second attempt at problem solving. 
A break in conscious work can facilitate problem solving during a second attempt. However, evidence for such incubation effects has been somewhat mixed (Olton, 1979; Smith \& Blakenship, 1989; Vul \& Pashler, 2007). There are various hypotheses that attempt to account for the mechanisms underlying incubation effects such as conscious work, opportunistic assimilation, and forgetting fixation. The conscious work hypothesis suggests that when an individual discontinues a focused effort on problem solving, they continue with more limited amounts of conscious work during the break. Upon secondary attempts, solving is improved due to the extra amount of conscious work during the break (Smith, 2011; Smith, Sifonis \& Angello, 2012). Other hypotheses focus primarily on the influence of unconscious processing during the interval between the initial and subsequent attempt. The opportunistic assimilation hypothesis suggests that when an individual is unable to solve a problem, the problem is tagged in the mind as unsolved. When an individual is not working on the problem, cues in the environment that fit the potential solution state can be picked up and compared to the unsolved problem. Correct cues that represent (or serve as a clue to) the solution are assimilated into the goal state and verified as the solution. This hypothesis suggests that individuals are unconsciously perceiving stimuli in the environment and relating the stimuli back to the representation of an unsolved problem (Seifert, Meyer, Davidson, Patalano, \& Yaniv, 1995). Lastly, the forgetting-fixation hypothesis suggests that incubation effects occur because the delay allows the decay of activation of incorrect solutions and solution paths. During the delay, the incorrect activation becomes less accessible and therefore "forgotten." Once they are forgotten, the individual can continue work on a more advantageous path after the delay (Smith \& Blakenship, 1989).

While various hypotheses attempt to account for the mechanisms underlying incubation effects, the most common assumption is that incubation is the result of continued unconscious processing during the break in conscious work (Moss, Kotovsky, \& Cagan, 2007). To our knowledge, no study has addressed whether indicators of subthreshold solution related activation during a failed attempt at problem solving predict which problems are more likely to be solved after an incubation period.

If unconscious solution-related activation contributes to both intuitive TOT judgments and incubation effects, intuitive TOT judgments may predict incubation in problem solving and the manner in which the solution is eventually generated. Unconscious processing is thought to contribute more to solving by insight than solving by analysis (Sternberg \& Davidson, 1995). During insight problem solving, solvers are unable to consciously report the processing that leads to overcoming the impasse, experience the solution as arising suddenly, and immediately recognize the correctness of the solution. Initial processing of an insight problem produces subthreshold activation of prepotent yet incorrect information, often resulting in a failed attempt (Simon, Newell, \& Shaw, 1979). Therefore, insight problem solving can likely benefit from an incubation period where the activation of 
incorrect information can diminish (e.g., Smith \& Blankenship, 1989; 1991) while solutionrelated activation is maintained, or when the activation of solution related information can grow stronger.

Taken together, intuitive TOT experiences may indicate subthreshold solution related activation and should occur for problems that are more likely to be solved after a delay in conscious work, thereby predicting incubation of problem solving. When an individual is unable to generate a solution to a problem, the solution may, nevertheless, have subthreshold activation. This activation may give rise to an intuitive TOT experience signaling to the individual that the solution is present, if inaccessible, in memory. The subthreshold solution related activation might persist during a break in conscious deliberation. Upon a second attempt at solving, this activation may break the conscious threshold and promote the solver's ability to generate the solution.

Moreover, the subthreshold solution related activation that gives rise to an intuitive TOT might specifically promote problem solving by insight. When previously subthreshold solution related activation breaks the conscious threshold, the solution may arise suddenly producing a moment of insight, while the solver remains unable to explicitly report the processing that contributed to their solution. When people perform a visual attention task that encourages them to attend to weakly activated information, they subsequently improve the number of verbal problems they solve, especially those they report solving with insight, suggesting that the ability to attend to or utilize subthreshold activation is malleable (Wegbreit, Suzuki, Grabowecky, Kounios \& Beeman, 2012).

This experiment investigated whether intuitive TOT judgments would predict improvements in solving when individuals try to solve word problems. We predicted that an intuitive TOT experience about the solution to an unsolved word problem would predict subsequent solving, specifically by insight, after a 24-hour incubation period.

\section{Methods}

\section{Participants}

Participants were native English speaking Northwestern University undergraduate volunteers, aged 18-22 years old, who participated in the experiment in exchange for course credit after signing informed consent. Fifty-seven participants completed the experiment, but data from eight participants were excluded from analyses due to being 2.5 standard deviations above or below the group mean on four dependent variables (i.e., the frequency of correct problems solved on Day 1 and on Day 2, i.e., people who did not solve these problems well; and the frequency of reporting a Tip of the Tongue or no Tip of the Tongue on Day 1, i.e., people for whom there were too few trials in one category or the other on Day 2). Thus, results include data from 49 participants.

- volume 4, no. 2 (Spring 2012) 


\section{Stimuli}

On Day 1, participants attempted to solve 96 Compound Remote Associate (CRA) problems (Bowden \& Jung-Beeman, 2003a), and on Day 2 attempted to solve 48 New problems and all of their unsolved Old problems from Day 1. CRA problems, a test of creative cognition, are patterned after the Remote Associates Test (RAT) (Mednick \& Mednick, 1967). Success on the RAT has been shown to correlate with success on classic insight problems, such as the nine-dot problem and the prisoner escape problem (Dallob \& Dominowski, 1993; Schooler \& Melcher, 1995).

CRA problems include three remotely related words (crab, pine, sauce). Solvers must think of one solution word (apple) that can form a common compound word or familiar two-word phrase with each of the three problem words (crabapple, pineapple, apple sauce). Reaching a solution requires solvers to think of more distantly related associates in order to connect each of the three problem words. CRA problems can be solved with a moment of insight, where solvers are unable to report how the solution came to their mind, or analytically, where solvers are able to consciously report how the solution came to their mind (Bowden \& Beeman, 2003b). Self-reports differentiating between insight and analytic solving have demonstrated reliable associations with numerous behavioral and neuroimaging markers (Bowden \& Jung-Beeman, 2003b; Jung-Beeman, et al., 2004; Kounios et al., 2006, 2008; Subramaniam, Kounios, Parrish, \& Jung-Beeman, 2009). Moreover, neurofeedback training differentially changes performance on problems reportedly solved by insight or solved analytically (Haarmann, Georgey, Smaliy, \& Dien, 2012).

\section{Procedure}

On Day 1, participants attempted to solve 96 problems, consisting of three problem words presented on three lines in the center of a computer screen for ten seconds. If participants were able to generate a solution, they stated the solution word aloud to the experimenter. Following each correct solution, participants reported how they solved the problem, either with insight or analytic processing. Detailed descriptions of both insight and analytic processes were described to ensure each participant was able to differentiate between the two problem solving styles (insight or analytic). Solving with insight was described as, "The answer suddenly comes to mind even though you are unable to articulate how you achieved the solution." Solving analytically was described as, "You deliberately and consciously tested out different words until you found the solution and you are able to report the steps that you used to reach the solution." Participants were informed that these were ideal descriptions, that they should choose which problem solving style most accurately described how they achieved the solution, and that no problem solving style was any better or any worse than the other. Participants had to judge each solution as insight or analytic, because in prior studies, if a third category "other" was available, some participants chose that too frequently. Participants were informed that performance on 
these problems did not measure intelligence, personality or mood and that they would not be able to solve every problem. Participants were only allowed to state one word aloud, and if they stated an incorrect solution, the problem was considered an unsolved problem.

If participants were unable to generate a solution, they indicated whether they experienced: a strong TOT ("The solution is stuck on the tip of your tongue"), a weak TOT ("You feel like you have some idea about what the solution could be"), or No TOT. Participants also indicated how easy they believed the problem would be for an average student to solve. These Ease ratings were made on a 5 -point Likert scale ( 1 =very easy to $5=$ very hard). The order of the TOT judgments and Ease ratings alternated throughout the experiment. Participants were instructed that TOT judgments were in reference to their particular experience regarding the solution, whereas the Ease ratings were in reference to the experience of a hypothetical problem solver (an average student). After the problem presentation and subsequent judgments, participants advanced to the next problem. Participants were allowed to report a solution to a problem at any time during the experiment, and problems solved after the time limit were excluded from further analyses.

At the end of testing on Day 1, participants were told they would receive a new set of problems on Day 2, and to not think about the problems anymore. At the start of Day 2 (i.e., $24+/-2$ hours), participants were asked if they had thought about the experiment since Day 1. No participant reported thinking about the experiment or solving any problems during the delay.

On Day 2, participants attempted to solve 48 new problems, randomly intermixed with their old problems. The Day 2 procedure was the same as the Day 1 procedure with the following exceptions: 1 ) In order to facilitate problem solving, participants were given 15 seconds, rather than 10 seconds, to solve each of the new and old problems. 2) When a participant correctly solved an old problem, after they rated whether they achieved the solution via a moment of insight or analytically, they again indicated whether they had thought of the answer while outside of the laboratory testing room. 3) If participants were unable to solve the problem, they were not asked to rate whether or not they experienced a TOT.

\section{Results}

\section{Day 1}

On Day 1, participants solved an average of 34 problems (35.4\%) within 10 seconds and an additional 3 problems (3.1\%) after the time limit. Because relatively few TOTs were reported, strong TOT and weak TOT reports were collapsed into one category (Yes-TOT). On average, 59 problems (61.5\%) remained unsolved, and participants reported experiencing a TOT (Yes-TOT) $(M=30, S D=12)$ on approximately the same number of problems 
for which they reported experiencing No TOT (No-TOT) $(M=29, S D=13), t(48)=0.29, p=$ .77. Due to different (and low) frequencies of problems assigned each rating by different participants, Ease ratings of 1, 2, and 3 were collapsed into an easy problem category and Ease ratings of 4 and 5 were collapsed into a hard problem category. Participants rated more unsolved problems as easy $(M=33, S D=15)$ compared to hard $(M=25, S D=14)$, $t(48)=2.09, p=.04$. TOT ratings did not reliably correlate with Ease ratings, $r(47)=.19$, n.s. That is, there was no reliable correlation between participants rating a problem as easy and also reporting a Yes-TOT for the same problem.

\section{Day 2}

Approximately 24 (+/- 2) hours later, participants returned to solve another set of problems, 48 new problems and an average of 59 old problems (i.e., Day 1 unsolved problems). The proportion of old problems that were solved at retest on Day 2 was defined as the Improvement Score (Smith \& Blakenship, 1991). On average, participants solved 9 (SD = 4 ) of 59 old problems, resulting in an average Improvement Score of $16 \%$.

\section{TOT Specific Incubation Effect}

In order to increase the number of observations per participant and compare solving proportions across participants, we analyzed the Day 2 TOT specific solving behavior of only the participants $(N=35)$ who on Day 1 had more than 15 old problems in both Yes-TOT and No-TOT categories. This filter removed participants with less than $25 \%$ of their total TOT responses in either Yes-TOT or No-TOT category. As predicted, participants solved a reliably greater percentage of old problems for which they experienced a TOT on Day 1 $(M=18 \%, S D=9 \%)$ than Old problems for which they experienced No-TOT $(M=15 \%$, $S D=9 \%), t(34)=2.74, p=.01$. However, participants did not reliably differ in solving old problems that they judged as easy $(M=16 \%, S D=9 \%)$ compared to problems that they judged as hard $(M=15 \%, S D=9 \%), t(48)=0.34, p=.73$.

Some participants may have been more sensitive to the unconscious activation thought to underlie TOT judgments, thus more precise in reporting TOT experiences. These participants would be more likely to demonstrate a TOT specific incubation effect than participants who were less sensitive to unconscious activation. Using a median split, we examined whether participants who were more successful after the incubation period (i.e., $N=17$, participants with an Improvement Score above the median) differed from participants who did not incubate well (i.e., $N=15$, participants with an Improvement Score below the median). Participants with Improvement Scores above the median solved a greater proportion of old problems for which they experienced a TOT on Day 1 $(M=22 \%, S D=6 \%)$ compared to problems for which they experienced No-TOT $(M=17 \%$, $S D=6 \%), t(16)=2.23, p=.04$. However, participants with Improvement Scores below 
the median did not reliably differ in solving old problems for which they experienced a TOT $(M=10 \%, S D=5 \%)$ compared to No-TOT $(M=10 \%, S D=5 \%), t(14)=0.16, p=.89$. That is, the participants who had the highest Improvement Scores demonstrated a TOT specific incubation effect that participants below the median Improvement Score did not demonstrate (see Figure 1). Here again, neither participant group (i.e., above or below the median Improvement Score) reliably differed in solving old problems that they judged as easy compared to problems that they judged as hard (all $t s<1.0$ ).

\section{TOT and Insight Solving}

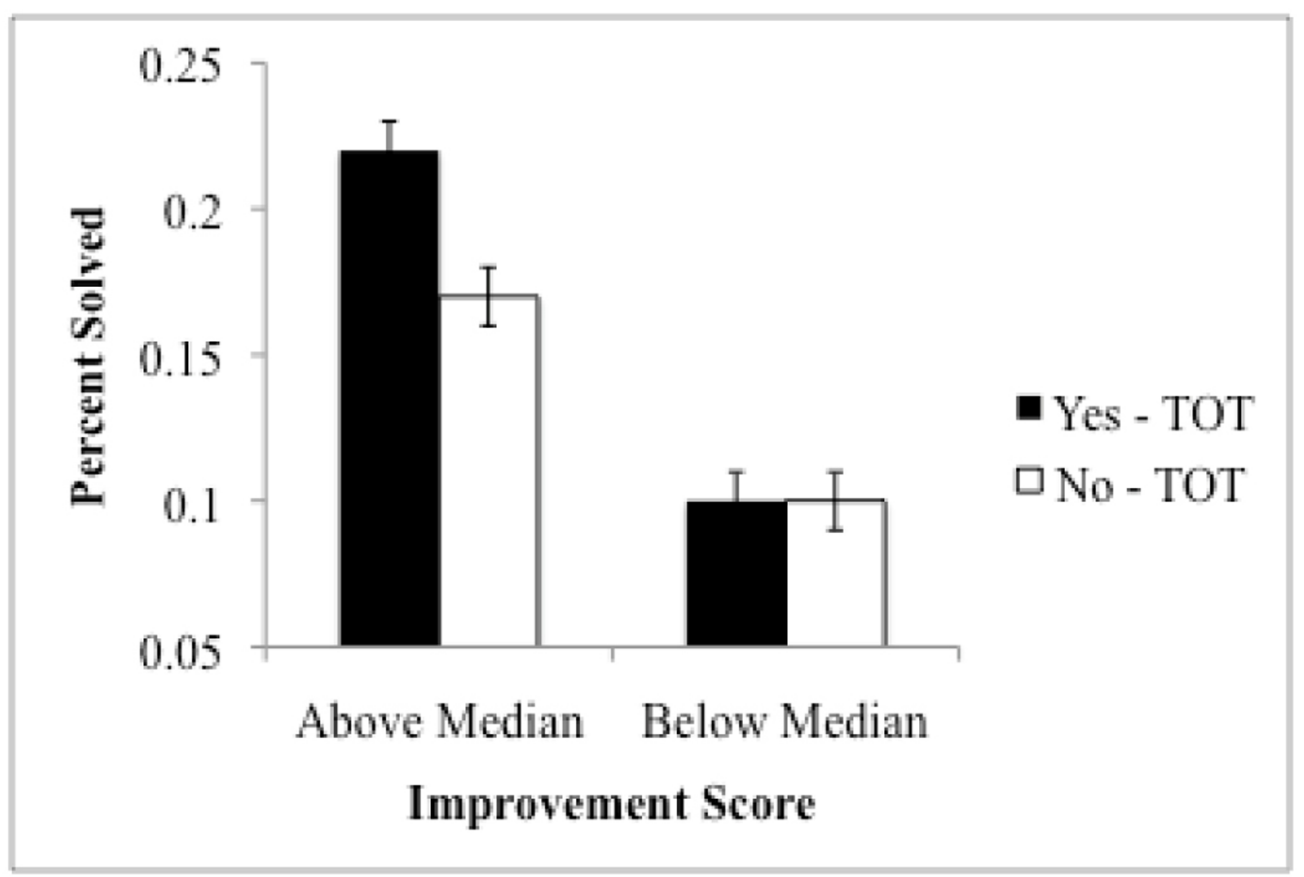

Figure 1. Percent of problems solved on Day 2 for which participants, above and below the median Improvement Score, experienced a Yes - TOT or a No - TOT on Day 1.

Intuitive TOT judgments seemed to interact with the way in which participants reported solving old problems (either with insight or analysis). Specifically, when participants were unable to generate a solution but experienced a TOT on Day 1, on Day 2 they were marginally more likely to report solving those problems with insight $(M=67 \%, S D=33 \%)$, compared to problems where they experienced No-TOT $(M=56 \%, S D=34 \%), t(48)=1.89$, $p=.06$ (see Figure 2). 


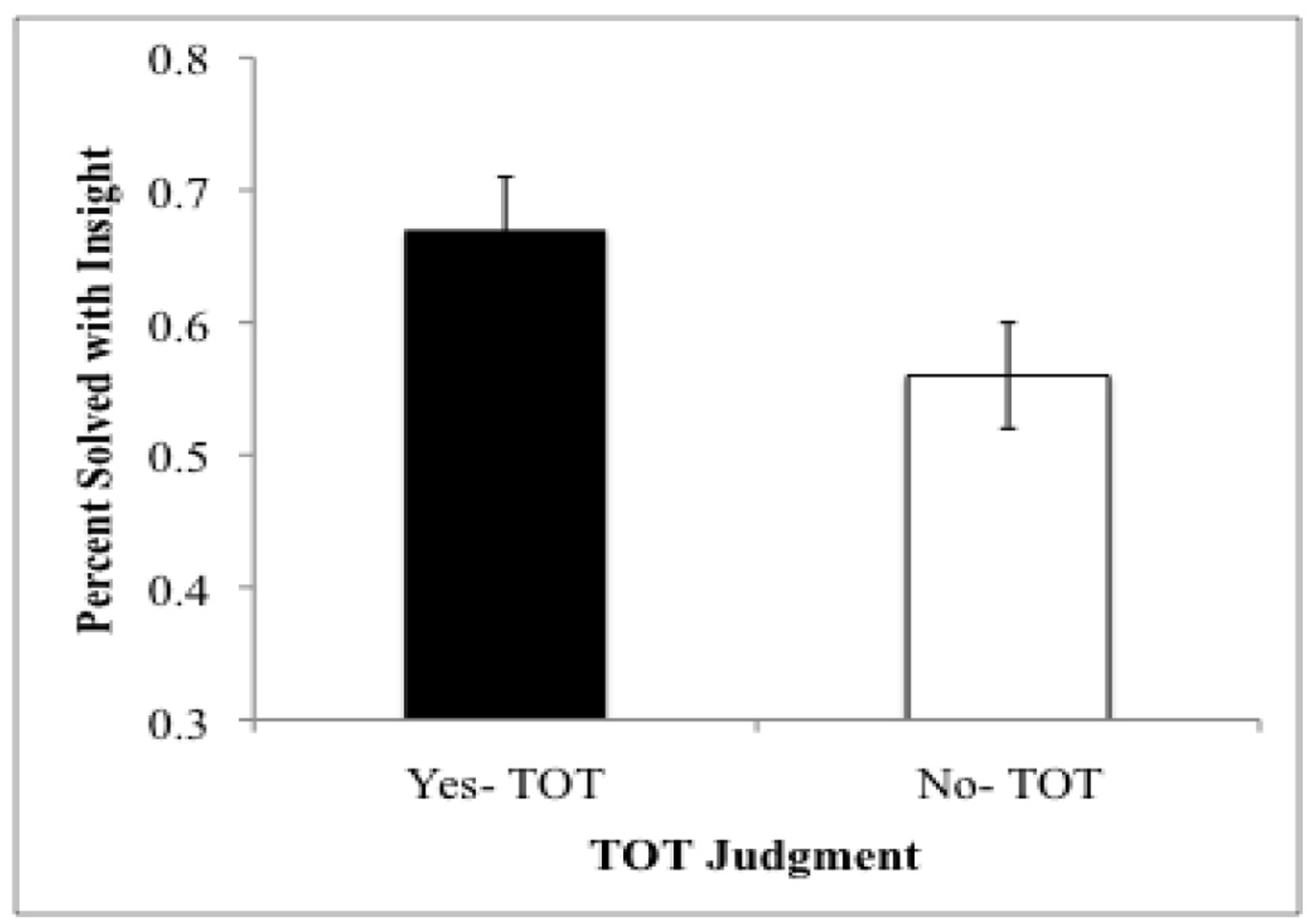

Figure 2. Percent of problems solved when participants reported solving with insight on Day 2 for which participants experienced a Yes - TOT or a No - TOT on Day 1.

\section{Discussion}

When individuals fail to solve a problem during an initial attempt, intuitive TOT judgments reliably predict subsequent solving after a 24-hour incubation period. Further, TOT experiences on Day 1 were related to reports of solving by insight on Day 2. When individuals were unable to generate the solution to a remote associate problem, but experienced a TOT (a sense that they were close, or that they had some solution related information in the back of their mind), they were more likely to solve those problems (compared to problems when they did not experience a TOT) after an overnight incubation period. Prior studies have indicated that sleep may facilitate memory reorganization (Ellenbogen, Payne, \& Stickgold, 2006) and specifically solutions by insight (Wagner, Gais, Haider, Verleger, \& Born, 2004). In addition to the TOT specific incubation effect, experiencing a TOT was associated with marginally more frequent reports of insight solving, demonstrating a potential relationship between intuition and insight. This experiment provides evidence that intuitive judgments about the problem solving process reliably indicate which problems are more likely to benefit from an incubation period, and to eventually be solved with a feeling of insight. Moreover, our results suggest that subthreshold solution 
related activation might underlie intuitive TOT judgments, incubation, and the experience of insight problem solving. Prior research has demonstrated that undetectable hints facilitate the solving of insight-like problems (Bowden, 1997).

Intuitive TOT judgments have been investigated in previous behavioral and neuroimaging studies to measure the dissociation between knowledge that one is subjectively aware of but unable to consciously retrieve. Behaviorally, when presented the definition of a low frequency word and asked to try to recall the word, participants that experienced a TOT were able to describe numerous features of the unrecalled word (i.e., some of the letters, the number of syllables, and the intonation) without being able to consciously retrieve the word (Brown \& McNeill, 1966, Koriat \& Leiblich, 1977). Neurally, when presented with a description of an esoteric proper noun and asked to retrieve the name, activation in the anterior cingulate and right middle frontal regions dissociated the intuitive TOT state from an explicit retrieval state. Increased activation in the prefrontal cortex is thought to reflect increased retrieval monitoring activity when retrieval failure results in a subjective feeling of partial recovery (Maril et al., 2001; 2005).

In the current study, a similar intuitive TOT judgment was used to assess an individual's subjective feeling of partial semantic activation of solution-related information after a failed attempt to solve a remote associate problem. When an individual attempts to solve a remote associate problem, activation of the three problem words spread to semantically related associates in semantic memory. This spreading activation eventually converges upon the common remote associate, which is the solution word. If the subthreshold activation of solution-related information is not sufficient to support conscious awareness of the solution, the individual is unable to solve the problem (Ilg et al., 2007). However, this subthreshold solution related activation might become relatively more accessible during incubation and subsequent attempts at solving (Connor, et al., 1992). The current study suggests that the subthreshold solution related activation can give rise to a valid intuitive TOT experience.

Research with anagrams, rebuses, and remote associate problems has demonstrated incubation effects after an incubation period as short as 1 minute to as long as 24 hours (Sio \& Ormerod, 2009). Prior studies have contrasted an incubation condition with a continued work condition. However, we were interested mainly in how intuitive TOT judgments would relate to incubation effects. Therefore, we assessed the specific differences between Yes- TOT and No-TOT reports on subsequent solving after an incubation period. Our results demonstrated that intuitive TOT judgments were sensitive, as they predicted which unsolved problems were more likely to be solved during a second attempt at problem solving, providing support for a TOT specific incubation effect. Regarding the mechanisms underlying incubation effects, there is less evidence supporting the continued conscious work hypothesis and stronger evidence in favor of the forgetting fixation hypothesis 
(Olton, 1979; Smith \& Blakenship, 1989; Vul \& Pashler, 2007). In the current study, we did not induce fixation, although it remains possible participants still experienced internal fixation. On the other hand, perhaps continued unconscious processing can occur better when there is a basis from which to work, like subthreshold solution related activation that we assessed with the TOT judgments.

It is unlikely that our TOT specific incubation effect was based on continued conscious work that was carried out on problems that elicited TOT experiences. Clearly, failure to solve any problem indicates a need for continued effort, but no participant reported consciously working on or solving any problems (No-TOT or Yes-TOT) during the incubation delay. Moreover, it is unlikely that our TOT specific incubation effect was based on intuitive TOT experiences indicating the problems that should be easily solvable. On Day 1 , for each unsolved problem, in addition to making a TOT judgment, participants also rated how easy they thought the problem was in general (i.e., for an average student). These Ease ratings were not predictive of Day 2 solving. That is, unsolved Day 1 problems that were rated as easy were no more likely to be solved on Day 2 compared to problems that were rated as hard. Moreover, there was no reliable correlation between participants rating a problem as easy and also reporting a Yes-TOT for the same problem. Thus, the TOT specific incubation effect was not due to perceived ease of problems. Rather, the intuitive TOT judgment was likely based on subthreshold solution related activation that was insufficient to support immediate solution, but sufficient to support some component of incubation. That is not to say that participants had absolutely no subthreshold solution related activation for problems when they did not experience a TOT, but if there was activation, it was insufficient to influence their intuitive judgment.

It remains possible that other mechanisms of incubation effects related to unconscious processing (i.e., opportunistic assimilation and forgetting fixation) may account for our results. Individuals may have assimilated solution related information during the incubation period. If unsolved problems that elicited a TOT experience reflected greater subthreshold solution related activation (relative to problems that elicited no TOT experience), it is plausible that during an incubation period these problems would have had an advantage in unconsciously assimilating cues from the environment that relate to the solution. However, the influence of opportunistic assimilation may have been negligible, considering the fact that no participant solved any problems during the incubation break. Given the problems are highly constrained (i.e., there is only one word that can fit with each problem word), the best "clue" would be the solution word itself, and would have led to solving.

According to the forgetting fixation hypothesis, incubation effects occur only when the critical misleading clues that led to the initial fixation are forgotten and other solution paths are explored. The incubation effects demonstrated in the majority of the aforemen- 
tioned studies only occurred when participants were presented with misleading clues that externally induced fixation (Olton, 1979; Smith \& Blankenship, 1989; Vul \& Pashler, 2007). We did not externally induce fixation during the presentation of the problems in this study, but participants might have experienced an internal fixation, from strong associations to problem words, that prevented conscious access to the solution. Fixation could influence the TOT judgments. Specifically, on some trials strong fixation could block access to the weak solution-related activation, whereas on trials without fixation, even weak solution-related activation can be accessed. Thus, weak solution activation without solving will occur only on trials for which there is fixation. Thus the TOT judgment may have been signaling the presence both of subthreshold solution activation and strong fixation. If the fixation decays or is forgotten during incubation, then these trials are more likely to be solved on Day 2, so long as activation related to solution is maintained. Thus, our data are compatible with fixation forgetting, and cannot completely determine the specific mechanism of incubation.

In addition to the TOT specific incubation effect, our results demonstrate that experiencing a TOT (compared to No-TOT) led to marginally greater reports of solving with insight, thereby demonstrating a potential relationship between intuition and insight. During a second attempt at solving, the subthreshold solution related activation that gave rise to an intuitive TOT judgment continued to summate until it broke the conscious threshold, marginally more often via a moment of insight compared to analytical processing. It seems that both intuition and insight reflect related phases of unconscious processing: intuition (in this case, the TOT judgment), as the subjective experience of subthreshold solution related activation, and insight as the sudden shift of unconscious knowledge into conscious awareness via an Aha! moment. Even though the bases of intuitions are not accessible for conscious reflection, our data suggest that intuitive TOT judgments can reliably indicate which unsolved problems will benefit from an incubation period and which unsolved problems are more likely to be reported as solved with insight during a second attempt.

\section{References}

Beeman, M., Friedman, R. B., Grafman, J., Perez, E., Diamond, S., \& Beadle Lindsay, M. (1994). Summation priming and coarse semantic coding in the right hemisphere. Journal of Cognitive Neuroscience, 6, 26-45.

Bolte, A., \& Goschke, T. (2005). On the speed of intuition: Intuitive judgments of semantic coherence under different response deadlines. Memory \& Cognition, 33 (7), 1248-1255.

Bolte, A., Goschke, T., \& Kuhl, J. (2003). Emotion and intuition: effects of positive and negative mood on implicit judgments of semantic coherence. Psychological Science, 14, 416-421.

- volume 4, no. 2 (Spring 2012) 
Bowden, E. M. (1997). The effect of reportable and unreportable hints on anagram solution and the aha! experience. Consciousness and Cognition, 6, 545-573.

Bowden, E. M., \& Beeman, M. J. (1998). Getting the right idea: Semantic activation in the right hemisphere may help solve insight problems. Psychological Science, 6, 435-440.

Bowden, E., \& Jung-Beeman, M. (2003). Normative data for 144 compound remote associate problems. Behavior Research Methods, Instruments \& Computers, 35(4) 634-639.

Bowers, K. S., Regehr, G., Balthazard, C., \& Parker, K. (1990). Intuition in the context of discovery. Cognitive Psychology, 22, 72-110.

Brown, R., \& McNeill, D. (1966). The "tip of the tongue" phenomenon. Journal of Verbal Learning and Verbal Behavior, 5, 325-337.

Connor, L., Balota, D. A., \& Neely, J. H. (1992). On the relation between feeling of knowing and lexical decision: persistent subthreshold activation or topic familiarity? Journal of Experimental Psychology: Learning, Memory, and Cognition, 18(3), 544-554.

Dallob, P. L., \& Dominowski, R. L. (1993). Erroneous solutions to verbal insight problems: Effects of highlighting critical material. Paper presented at the meeting of the Western Psychological Association, April.

Dorfman, J., Shames, V. A., \& Kihlstrom, J. F. (1996). Intuition, incubation, and insight: implicit cognition in problem solving. In Underwood, G. (Eds.), Implicit cognition (pp. 257-296). Oxford Univ. Press.

Ellenbogen, J. M., Payne, J. D., \& Stickgold, R. (2006). The role of sleep in declarative memory consolidation: passive, permissive, active or none? Current Opinion in Neurobiology, 16, 1-7.

Epstein, S., Pacini, R., Denes-Raj, V., \& Heier, H. (1996). Individual differences in intuitiveexperiential and analytical-rational thinking styles. Journal of Personality and Social Psychology, 71(2), 390-405.

Haarmann, H. J., George, T., Smaliy, A., \& Dien, J. (2012). Remote associates test and alpha brain waves. The Journal of Problem Solving, 4(2), Article 4.

Ilg, R., Vogeley, K., Goschke, T., Bolte, A., Shah, J., Poppel, E., \& Gereon, F. (2007). Neural processes underlying intuitive coherence judgments as revealed by $\mathrm{fMRI}$ on a semantic judgment task. Neurolmage, 38, 228-238.

Jung-Beeman, M., Bowden, E. M., Haberman, J., Frymiare, J. L., Arambel-Liu, S., Greenblatt, R., Reber, P. J., \& Kounios, J. (2004). Neural Activity When People Solve Verbal Problems with Insight. PLoS Biology, 2(4): e97.

Kahneman, D. (2003). A perspective on judgment and choice: Mapping bounded rationality. American Psychologist, 58, 697-720.

Kahneman, D., Slovic, P., \& Tversky, A. (1982). Judgment under uncertainty: Heuristics and biases. New York: Cambridge University Press.

Koriat, A. \& Leiblich, I. (1977). What does a person in a "tot" know that a person in a "don't know" state doesn't know. Memory \& Cognition, 2, 647-755. 
Kounios, J., Fleck, J. I., Green, D. L., Payne, L., Stevenson, J. L., Bowden, E. M., \& Jung-Beeman, M. (2008). The origins of insight in resting-state brain activity. Neuropsychologia, 46 (1), 281-291.

Kounios, J., Frymiare, J. L., Bowden, E. M., Fleck, J. I., Subramaniam, K., Parrish, T. B., \& JungBeeman, M. (2006). The prepared mind: neural activity prior to problem presentation predicts subsequent solution by sudden insight. Psychological Science, 17 (10), 882890.

Liberman, M. D. (2000). Intuition: A social cognitive neuroscience approach. Psychological Bulletin, 126, 109-137.

Maril, A., Simons, J. S., Weaver, J. J., \& Schacter, D. L. (2005). Graded recall success: An event related fMRI comparison of tip of the tongue and feeling of knowing. Neurolmage, 24(4), 1130-1138.

Maril, A., Wagner, A. D., \& Schacter, D. L., (2001). On the tip of the tongue: an event-related fMRI study of semantic retrieval failure and cognitive conflict. Neuron, 31, 653-660.

Mednick, S. A., \& Mednick, M. T. (1967). Remote associates test: Examiner's manual. College and adult forms 1 and 2. Boston: Houghton Mifflin.

Moss, J., Kotovsky, K., \& Cagan, J. (2007). The influence of open goals on the acquisition of problem-solving information. Journal of Experimental Psychology: Learning, Memory, and Cognition, 33(5), 876-891.

Newell, A., \& Simon, H. A. (1972). Human problem solving. Englewood Cliffs, NJ: Prentice-Hall.

Olton, R. M. (1979). Experimental studies of incubation: Searching for the elusive. Journal of Creative Behavior, 13, 9-22.

Schooler, J. W. \& Melcher, J. (1995). The ineffability of insight. In S. M. Smith, T. B. Ward, \& R. A. Finke (Eds.) The creative cognition approach (pp. 249-268). Cambridge, MA: The MIT Press.

Schwartz, B. (1999). Sparkling at the end of the tongue: The etiology of tip of the tongue phenomenology. Psychonomic Bulletin \& Review, 6 (3), 379-393.

Schwartz, B. L. (2011). The effect of being in a tip-of-the-tongue state on subsequent items. Memory \& Cognition, 39, 245-250.

Seifert, C. M., \& Patalano, A. L. (1991). Memory for incomplete tasks: A re-examination of the Zeigarnik effect. In Proceedings of the Thirteenth Annual Conference of the Cognitive Science Society. (pp.114-119). Chicago, IL.

Seifert, C. M., Meyer, D. E., Davidson, N., Patalano, A. L., \& Yaniv, I. (1995). Demystification of cognitive insight: Opportunistic assimilation and the prepared-mind perspective. In R. J. Sternberg \& J. E. Davidson (Eds.) The nature of insight. Cambridge, MA:The MIT Press.

Simon, H. A., Newell, A., \& Shaw, J. C. (1979). The process of creative thinking. In H. A. Simon (Ed.), Models of thought (pp. 144-174). New Haven: Yale University Press.

Sio, U. N., \& Ormerod, T. C. (2009). Does incubation enhance problems solving? A metaanalytic review. Psychological Bulletin, 135 (1), 94-120.

- volume 4, no. 2 (Spring 2012) 
Smith, S. M. (2011). Incubation. In M. A. Runco \& S. R. Pritzker (Eds.), Encyclopedia of creativity volume I, 2nd edition, (pp. 653-657). Academic Press.

Smith, S. M., \& Blankenship, S. E. (1989). Incubation effects. Bulletin of the Psychonomic Society, 27, 311-314.

Smith, S. M., \& Blankenship, S. E. (1991). Incubation and the persistence of fixation in problem solving. American Journal of Psychology, 104 (1), 61-87.

Smith, S. M., Sifonis, C. M., \& Angello, G. (2012). Clue insensitivity in remote associates test problem solving. The Journal of Problem Solving, 4(2), Article 2.

Sternberg, R. J., \& Davidson, J. E. (1995). The nature of insight. Cambridge, MA:The MIT Press.

Subramaniam, K., Kounios, J., Parrish, T. B., \& Jung-Beeman, M. (2009). A brain mechanism for facilitation of insight by positive affect. Journal of Cognitive Neuroscience, 21(3), 415-432.

Vul, E., \& Pashler, H. (2007). Incubation benefits only after people have been misdirected. Memory \& Cognition, 35, 701-710.

Wagner, U., Gais, S., Haider, H., Verleger, R., \& Born, J. (2004). Sleep inspires insight. Nature, 427 (6972), 352-355.

Wegbreit, E., Suzuki, S., Grabowecky, M., Kounios, J., \& Beeman, M. (2012). Visual attention modulates insight versus analytic solving of verbal problems. The Journal of Problem Solving, 4(2), Article 5.

Yaniv, I., \& Meyer, D. E. (1987). Activation and metacognition of inaccessible stored information: Potential bases for incubation effects in problem solving. Journal of Experimental Psychology: Learning, Memory, \& Cognition, 13, 187-205.

\section{Acknowledgments}

The authors would like to acknowledge Ezra Wegbreit for his contributions to this research, and the support of grant \#24467 from the John Templeton Foundation to MB. 